Apidologie, 1976, 7 (1) 85-104.

\title{
POLLINISATION DU CARTHAME (CARTHAMUS TINCTORIUS L.) \\ SES EFFETS SUR LA FORMATION ET LA GERMINATION DES SEMENCES
}

\section{Die Bestäubung des Echten Saflor (Carthamus tinctorius L.) und ihr Einfluss auf Samenbildung und Keimen.}

\section{E. BARBIER et M. NADIR}

avec la collaboration technique de J. M. Fonentiv et M. Slama.

Station expérimentale d'Apiculture Centre de Recherches d'Avignon, I.N.R.A., 84140 Montfavet

S.A.S.M.A. Casablanca - Maroc

\section{SUMMARY}

\author{
POLliNATION OF SAFFlower (Carthamus tinctorius L.). \\ ITS EFFECTS ON SEED SETTING AND SEED GERMINATION
}

In the safflower seed setting may occur naturally by autopollination, but it is known that the outcome of allopollination caused by insects' visits on flowers, especially those of honeybees, is setting of more seeds of which size and weight are smaller and oil content higher.

Allogamy provides also a germination energy much greater without changing the ultimate germination rate or germination capacity.

\section{RÉSUMÉ}

Chez le carthame, la formation des semences peut se produire naturellement par autopollinisation, mais il est constaté que l'allopollinisation provoquée par la visite des insectes sur les fleurs et plus particulièrement par celle des abeilles, a pour conséquence la formation de semences plus nombreuses, de taille et de poids réduits, et en même temps de teneur en huile plus élevée.

L'allogamie confère aussi aux semences une énergie germinative nettement plus grande sans pour autant modifier le taux final de germination ou faculté germinative. 


\section{INTRODUCTION}

Au lendemain de la deuxième guerre mondiale, la pénurie générale des matières grasses alimentaires a provoqué dans divers pays la mise en culture du carthame dont l'huile, connue pour avoir une teneur très élevée en acides gras insaturés, possède du fait de cette particularité des possibilités étendues d'utilisation.

Au Maroc, la production a atteint 25000 quintaux en 1950 mais la culture a régressé par la suite dans des proportions importantes. Depuis quelques années on constate un renouveau d'intérêt pour le carthame qui, pouvant végéter dans des zones à faible pluviométrie, là où les autres oléagineux et particulièrement le tournesol ont une production aléatoire, peut assurer un complément substantiel de matières grasses face à la progression constante des besoins.

Le carthame (Carthamus tinctorius L.) est une plante annuelle de la famille des composées. La racine pivotante, qui s'enfonce profondément dans le sol, permet à la plante de résister à la sécheresse. En culture la tige peut atteindre $1,50 \mathrm{~m}$; elle est ramifiée au sommet et chaque ramification est terminée par un capitule; le nombre des capitules est d'environ 30 par plante, mais il atteint parfois 60 et même 100 . Le capitule contient en moyenne 40 fleurs avec un maximum de 70 . L'involucre est constitué de bractées épineuses ou inermes selon les variétés, les variétés à bractées inermes sont reconnues plus sensibles aux attaques des moineaux.

Compte tenu de l'intérêt économique présenté par la culture du carthame, des essais ont été entrepris en 1965 sur la variété “ Gila " puis, avec plus de détails en 1969, sur la variété « S V 12 », pour examiner les effets que pouvait avoir le butinage des abeilles sur la formation des graines et sur la production d'huile de la plante $\left(^{1}\right)$. Les qualités germinatives des semences ont également été étudiées.

\section{I. - TECHNIQUES}

\section{A. - Essais aux champs}

Pour chaque essai, dans une zone de la culture où la végétation est très régulière, trois surfaces identiques sont déterminées. La première, dont les plantes se trouvent dans les conditions normales de milieu, constitue un lot de comparaison de plein air. Les deux autres surfaces sont identiques et sont recouvertes, juste avant la floraison, par des cages grillagées destinées à protéger les fleurs de la visite des insectes floricoles. Ces cages sont constituées par des cadres Meknès.

(1). « Gila » variété à bractées épineuses, cultivée à la Station expérimentale d'Ain Taoujdate près de

«S V 12 "Variété à bractées inermes, cultivée à Berrechid près de Casablanca dans le cadre du projet P.R.A.M. 
mesurant $1 \mathrm{~m} 50$ sur $3 \mathrm{~m}$, tendus de toile métallique dite de moustiquaire; les montants des cadres sont en hois, leur section carrée mesure $4 \mathrm{~cm}$ de côté. Un système d'assemblage par boulons permet, selon les besoins, la construction de cages cubiques, de tunnels ou de cages de grande surface. Dans le cas présent les cages mesurent $3 \mathrm{~m}$ sur $12 \mathrm{~m}$.

Au moment du montage des cages les plantes sont traitées à l'aide d'un insecticide à faible rémanence et, deux jours après, une ruchette contenant une petite colonie d'abeilles est introduite sous une des cages.

Pour plus de facilité les lots seront par la suite désignés par les symboles : PA (plein air), - Ab (sous cage sans abeilles), + Ab (sous cage avec abeilles).

\section{B. - Récolle et examen des semences}

Les plantes sont récoltées à maturité des semences.

Pour la variété a Gila w les semences sont extraites par battage d'un lot de 100 plantes. Les productions étant mélangées la portée des investigations se trouve de ce fait sensiblement limitée.

Pour la variété a SV 12 » les plantes sont conservées entières après la récolte. Ultérieurement dans chaque lot on procède à la constitution d'une série étroitement comparable aux autres. 40 plantes sont choisies dans le lot + Ab, elles sont mesurées et leurs capitules dénombrés. Dans les deux autres lots une série similaire est constituée c'est-à-dire que les plantes homologues sont rigoureusement semblables en taille et en nombre de capitules.

Par la suite la production de chaque capitule est pesée globalement et les semences sont dénombrées. Une semence est considérée comme normale lorsqu'elle est pesante, développée et renflée (même si elle est double); les semences dites avortées comprennent toutes les autres formes, même les ovaires non développés. Une pression sous le doigt ou le choc sur une surface dure permet le classement des semences douteuses.

\section{C. - Dosage des matières grasses}

Le dosage des matières grasses est effectué après broyage fin des graines; un prélèvement compris entre 5 à $6 \mathrm{~g}$ est pesé avec précision et introduit dans un Soxhlet. L'extraction des matières grasses est faite par l'éther de pétrole et la teneur en huile est exprimée par rapport à la matière sèche.

Pour la variété “ Gila », un échantillon de 50 g est prélevé dans la masse homogénéisée des graines produites par chaque lot. L'échantillon est suffisamment important pour effectuer plusieurs dosages (la moyenne seule est utilisée) et pour déterminer la teneur en eau.

Pour la variété “ SV 12 » les teneurs en huile sont déterminées sur les graines produites par chacune des plantes des trois séries. En règle générale la teneur en huile indiquée pour une plante est la moyenne de deux dosages. Cette teneur est retenue si l'écart entre les deux dosages ne dépasse pas 0,8 (4 plantes sur 119 ont été éliminées), mais en moyenne les écarts se situent aux environs de 0,3 .

Quant à la teneur en eau, elle est dosée sur des graines provenant des autres plantes de chaque lot et conservées dans les mêmes conditions que la série correspondante.

\section{D. - Germination des semences}

La germination des semences est obtenue sur des plateaux en aluminium mesurant $35 \times 22 \mathrm{~cm}$, dont le fond est occupé par un tapis absorbant genre a Spontex 》 de $1 \mathrm{~cm}$ d'épaisseur utilisé normalement pour les essais de germination. Le tapis est recouvert d'un tissu feutré et d'un papier buvard gris sur lequel il est plus facile de distinguer les germinations. Le tout est recouvert par le rabat en tissu feutré.

Le plateau est noyé dans l'eau pendant 30 minutes, puis renversé pendant quelques minutes pour éliminer l'excès d'eau. Le plateau est alors introduit dans une étuve biologique de $70 \mathrm{dm}^{3}$ de capacité réglée à la température désirée. On y ajoute un large cristallisoir contenant de l'eau et l'étuve est elle-même installée dans une pièce climatisée réglée à la même température que l'étuve et à humidité relative voisine de 100 pour cent. Les essais sont effectués aux températures de $11,14,5,17,5$ et $25^{\circ}$.

Après 24 heures la température du plateau s'est égalisée avec celle de l'étuve et l'atmosphère de celle-ci a acquis une humidité proche de la saturation. A partir de ce moment les 
semences sont disposées en lignes régulières sur le papier buvard. Seules les semences moyennes correspondant aux cribles $n^{0} 18,19$ et 20 sont utilisées dans ces essais, le diamètre des cribles étant 3,50, 3,75 et 4,00 millimètres. L'ensemencement d'un plateau se fait avec 100 semences du lot $+\mathrm{Ab}$ et $100 \mathrm{du}$ lot - $\mathrm{Ab}$. Chaque essai, répété 3 à 5 fois, porte sur un total de 600 à 1000 semences.

Le dénombrement des germinations est régulièrement effectué toutes les heures, sauf pour les essais à $11^{\circ}$ où après la vingt sixième heure ils sont faits toutes les 2 heures puis toutes les 3 et 4 heures. Le critère de germination retenu est l'écartement des téguments. C'est la première manifestation apparente de la germination et c'est d'ailleurs la plus facile à observer.

\section{II. - RÉSULTATS}

\section{A. - Aspect de la floraison}

Comme cela a été constaté sur d'autres plantes : Lavande, Clémentier et Tournesol (BARBier 1962, 1964 et 1966), la floraison des lots -Ab apparaît comme particulièrement abondante. Cela est dû au fait que les corolles restent épanouies pendant une semaine environ en gardant une belle couleur jaunecitron; elles ne fanent ensuite que lentement en virant progressivement au brun-rougeâtre; pendant toute cette période le pollen reste sur la colonne staminale.

Par contre chez les plantes visitées par les insectes, le pollen avidement récolté par les butineuses, n'est visible que pendant un court instant après l'anthèse; quant aux corolles elles ne restent épanouies que pendant 24 à 48 heures au plus et virent très rapidement au brun-rougeâtre, de ce fait les capitules ne portent jamais que quelques fleurs épanouies, 6 à 8 au plus, contre 30 à 35 sur ceux des plantes du lot $-\mathrm{Ab}$.

\section{B. - Production des semences}

Les premières observations faites sur la variété “ Gila » portent sur la récolte globale de 100 plantes, alors que pour la variété c SV 12 w la production de chaque plante a été analysée.

\section{Production globale}

Le tableau 1 permet de constater que chez le carthame (variété “ Gila ») l'autopollinisation et l'autofécondation autorisent la formation de nombreuses semences; par rapport au lot + Ab le rapport de production est de $76 \%$. Mais on doit remarquer que l'allogamie, provoquée par les abeilles, permet la formation de semences plus nombreuses et à teneur en huile plus élevée. Par contre le poids moyen des semences est plus élevé en autogamie qu'en allogamie.

Malgré cette dernière particularité le poids des récoltes et la production d'huile sont plus élevés pour le lot en pollinisation entomophile. 
TaвL. 1. - Caractéristiques des lots expérimentaux de carthame de la variété « Gila» TAв. 1. - Daten der Saflor-Versuchsparzellen (Varietät « Gila ")

\begin{tabular}{|c|c|c|c|c|}
\hline & & $-\mathbf{A b}$ & $+\mathbf{A b}$ & PA \\
\hline 1 & $\begin{array}{l}\text { Nombre de plantes dans le lot } \ldots \ldots \ldots \ldots \ldots \ldots \ldots \ldots \\
\text { Anzahl Pflanzen in der Parzelle }\end{array}$ & 100 & 100 & 100 \\
\hline 2 & $\begin{array}{l}\text { Poids des semences normales (en g) } \ldots \ldots \ldots \ldots \ldots \ldots \\
\text { Gewicht der normalen Samen (in } g \text { ) }\end{array}$ & 7320 & 9610 & 8970 \\
\hline 3 & $\begin{array}{l}\text { Rapport de production }(+\mathbf{A b}=100) \ldots \ldots \ldots \ldots \ldots \\
\text { Ertragsverhältnis }(+\mathbf{A b}=100)\end{array}$ & 76,2 & 100 & 91,5 \\
\hline 4 & $\begin{array}{l}\text { Poids moyen d'une semence (en } \mathrm{mg} \text { ) } \ldots \ldots \ldots \ldots \ldots \ldots \\
\text { Durchschnittsgewicht eines Sumenkorns (in } \mathrm{mg} \text { ) }\end{array}$ & 65,78 & 49,23 & 53,96 \\
\hline 5 & $\begin{array}{l}\text { Nombre de semences dans } 1000 \mathrm{~g} \ldots \ldots \ldots \ldots \ldots \ldots \\
\text { Anzahl Samen auf } 1000 \mathrm{~g}\end{array}$ & 15202 & 20312 & 18532 \\
\hline 6 & $\begin{array}{l}\text { Teneur en huile (en } \% \text { de la matière sèche) } \ldots \ldots \ldots \ldots \\
\text { Oelgehalt (in } \% \text { der Trockenmasse) }\end{array}$ & 38,00 & 46,12 & 42,15 \\
\hline 7 & $\begin{array}{l}\text { Poids d'huile pour le lot (en } g) \ldots \ldots \ldots \ldots \ldots \ldots \ldots \ldots \\
\text { Oelgewicht für die Parzelle (in g) }\end{array}$ & 2286 & 3602 & 3085 \\
\hline
\end{tabular}

- $\mathbf{A b}=$ sous cage sans abeilles.

$+\mathbf{A b}=$ sous cage avec abeilles.

$\mathbf{P A}=$ plein air.

- $\mathrm{Ab}=$ unter Drahtgeflecht ohne Bienen.

$+\mathbf{A b}=$ unter Drahtgeflecht mit Bienen.

PA $=$ im Freien, ohne Drahtgeflecht.

\section{$2^{\circ}$ Semences dans les capitules}

Pour la variété “ SV 12 ) les 3 séries présentent chacune 1059 capitules et chaque plante homologue en porte le même nombre, ou un nombre extrêmement voisin. Ces 3 séries sont donc comparables et les différences qui sont constatées ne peuvent être attribuéées qu'au facteur pollinisation.

Dans le tableau 2 on peut remarquer mieux encore que pour la variété (Cila », les incidences de l'allopollinisation sur la formation des semences. Pour le lot -Ab en effet :

- le taux d'avortement est nettement plus élevé

- le nombre de semences normales par capitule est plus faible

- le poids moyen d'une semence est plus élevé.

Il convient de remarquer encore que le carthame présente en même temps qu'une forte autofertilité un taux d'avortement toujours élevé quel que soit le mode de pollinisation. 
Tавц. 2. - Caractéristiques des lots expérimentaux de carthame de la variété «SV I2» Valeurs obtenues à partir des données individuelles

TAB. 2. - Daten der Saflor-Versuchsparzellen (Varietät “SV 12 »)

(Die Werte sind von Einzelangaben ausgehend gewonnen)

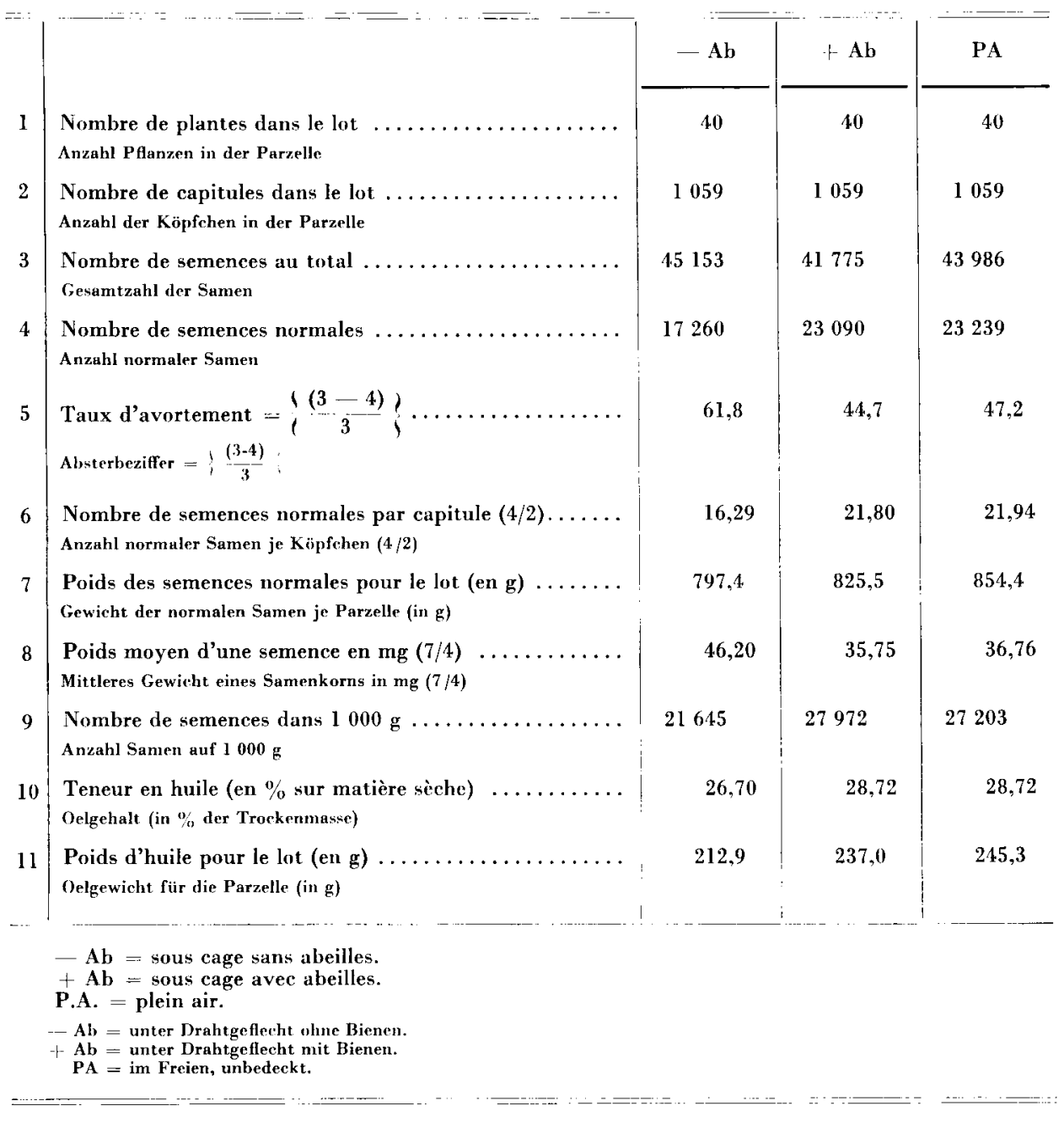

\section{$3^{\circ}$ Poids moyen des semences dans les capitules}

La figure 1 indique pour chaque lot la répartition des capitules selon le poids moyen de leurs semences. Les poids moyens couvrent 33 classes progressant régulièrement de $2,675 \mathrm{mg}$. La première classe comprend les semences pesant au minimum $6,43 \mathrm{mg}$ et la dernière celles pesant au maximum $94,70 \mathrm{mg}$. 
n. Capitules

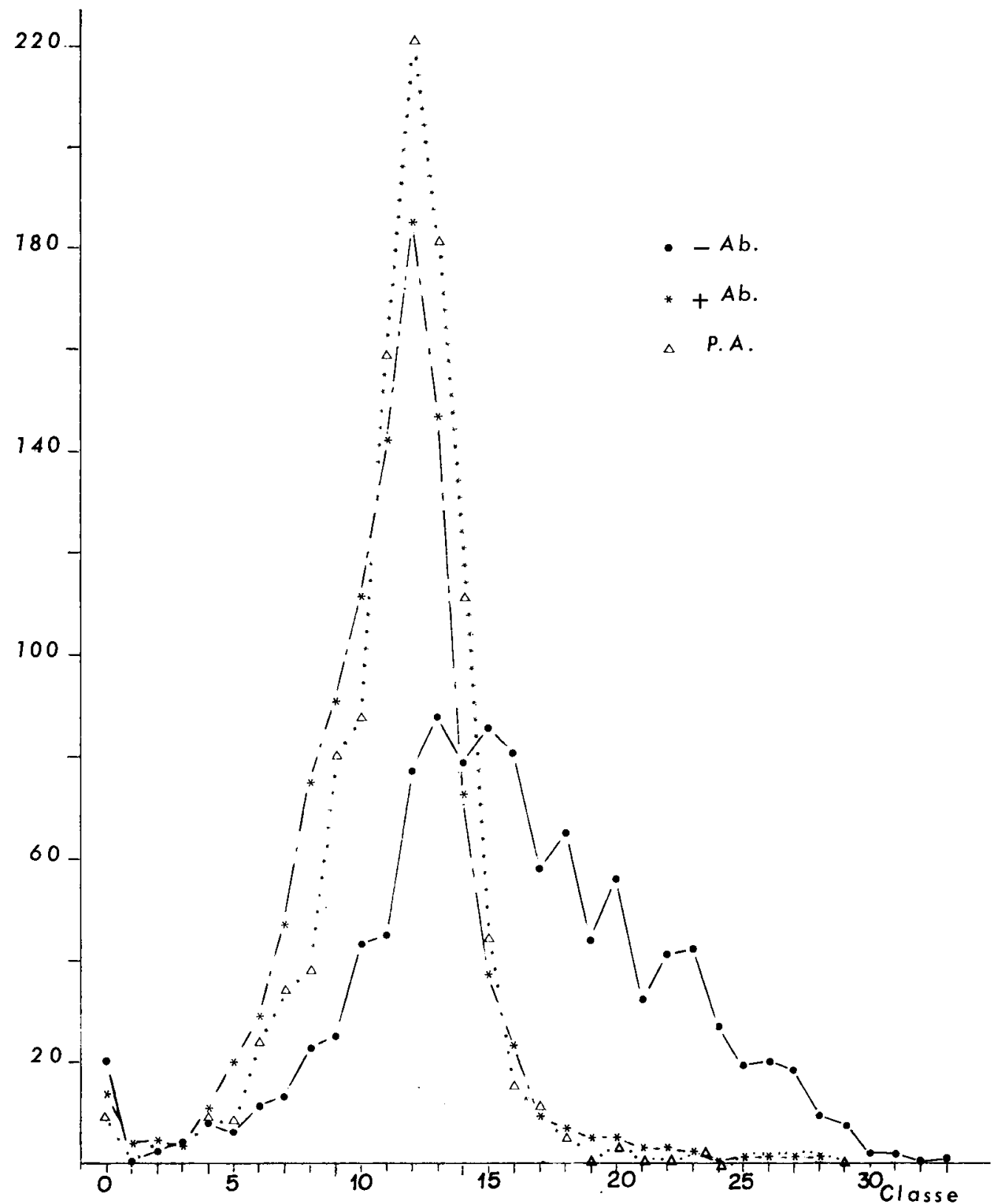

Fig. 1. - Distribution des capitules en fonction du poids moyen de leurs semences (Variêtés « SV $12 »$ ). Aв8. 1. - Verteilung der Köpfchen in Abhängigkeit von den Durchschnittsgewichten ihrer Samen (Varietät " SV 12 ") 
Les courbes des lots $+\mathrm{Ab}$ et $\mathrm{PA}$ sont très voisines, elles se caractérisent par un pic étroit très élevé en classe 13 (semences dont le poids moyen est compris entre 38,52 et $41,19 \mathrm{mg}$ ). Dans leur ensemble les semences de ces lots ne dépassent pas la classe 17 .

La courbe du lot -Ab par contre se caractérise par un sommet émoussé et has en classe 15 (poids moyen compris entre 43,87 et $46,54 \mathrm{mg}$ ) et par un net glissement vers les poids élevés.

\section{C. - Teneurs en huile}

Les teneurs en huile rapportées aux poids de la matière sèche, voir tableaux 1 et 2, montrent bien que les lots pollinisés par les abeilles ( $+\mathrm{Ab}$ et PA) possèdent des teneurs en huile supérieures à celles des lots -Ab et que les poids moyens des semences calculés pour les plantes entières sont plus faibles.

Cette double et inverse opposition apparaît plus nettement sur la figure 2 où les teneurs en huile déterminées sur les productions individuelles de chacune des plantes des trois séries, sont portées en fonction du poids moyen des semences de la plante. On remarquera que les plantes des lots $+\mathrm{Ab}$ et PA sont groupées avec une certaine densité dans les fortes teneurs en huile et les faibles poids moyens des semences, les moyennes générales étant respectivement 28,72 et $28,72 \%$ pour 35,75 et $36,76 \mathrm{mg}$; alors que les plantes du lot - Ab ont une répartition dispersée dans les faibles teneurs en huile et les poids élevés avec les moyennes générales de $26,70 \%$ et $46,20 \mathrm{mg}$.

\section{D. - Germination des semences}

Dans les essais de germination, les répétitions fournissent des résultats si voisins qu'il n'a pas été jugé utile de multiplier les figures. De préférence aux courbes nous avons choisi de porter à la figure 3 les relevés réels d'un essai pour chaque température. Par contre la figure 4 est construite à partir des courbes tracées d'après les moyennes.

Dans toutes les répétitions et d'une façon constante on peut remarquer (fig. 3) que :

a) la germination débute et évolue d'autant plus rapidement que la température est plus élevée,

b) les semences des lots $+\mathrm{Ab}$ germent toujours les premières.

c) dans les lots $+\mathrm{Ab}$ et - Ab les semences présentent la même capacité de germination et en fin d'opération elles atteignent les mêmes taux.

d) la pente des courbes de germination qui matérialise l'énergie germinative, est tjours plus forte pour les lots $+\mathrm{Ab}$ que pour les lots $-\mathrm{Ab}$.

Par ailleurs la comparaison des germinations telle qu'elle est exposée à la figure 4 , permet de constater que les écarts, entre les taux de germination 


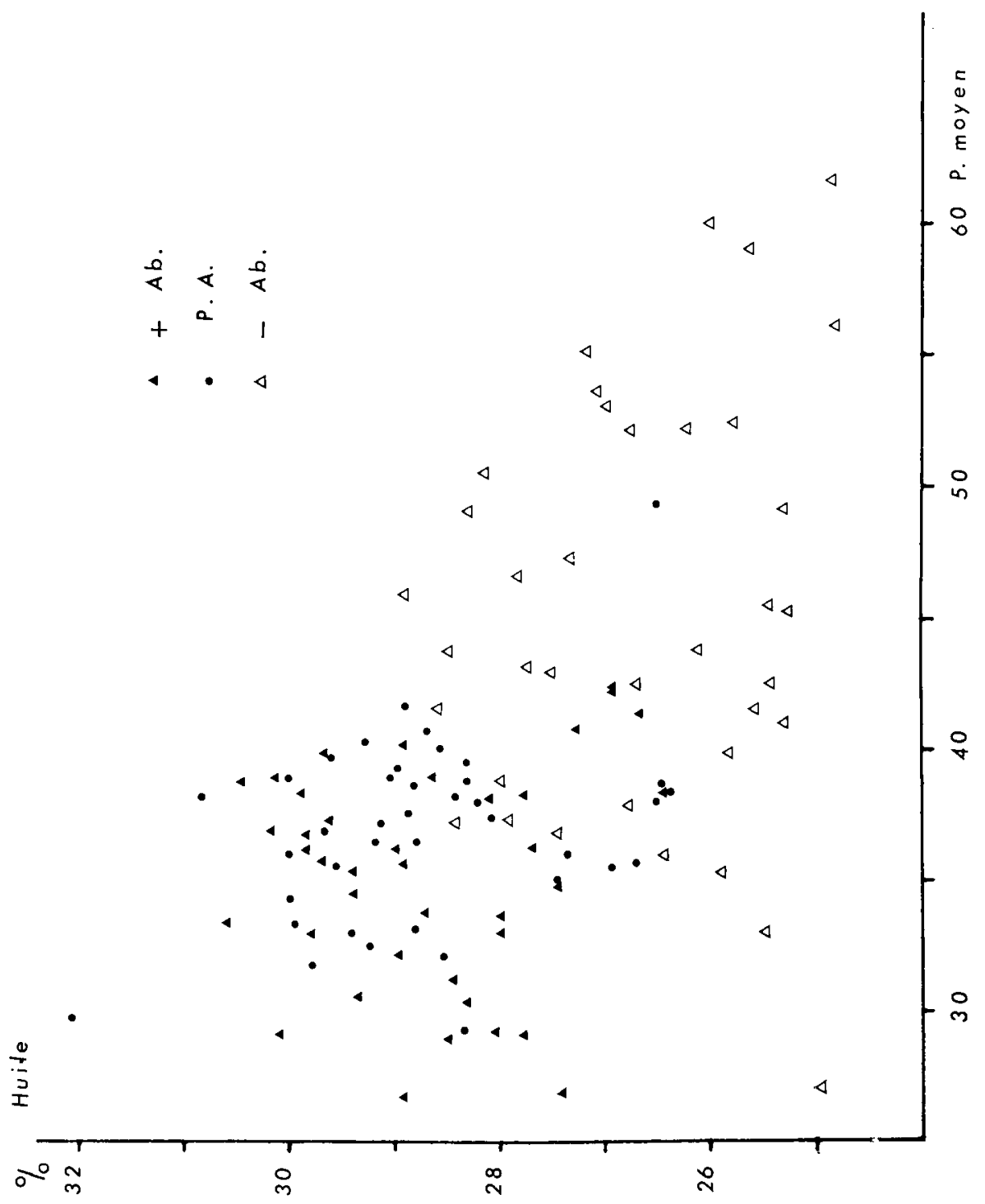

FIG. 2. - Répartition des teneurs en huile en fonction du poids moyen des semences de la plante (Variété « SV $12 »)$

Lot + Ab : 39 plantes; Lot - Ab : 37 plantes; Lot P.A : 39 plantes

Авв. 2. -- Verteilung des Oelgehaltes nach dem Durchschnittsgewicht der Samen der Pflanze (Varietät "SV 12 ")

Parzelle $+\mathbf{A b}=39$ Pflanzen.

Parzelle P.A. = 39 Pflanzen.

Parzelle $-\mathbf{A b}=37$ Pflanzen. 


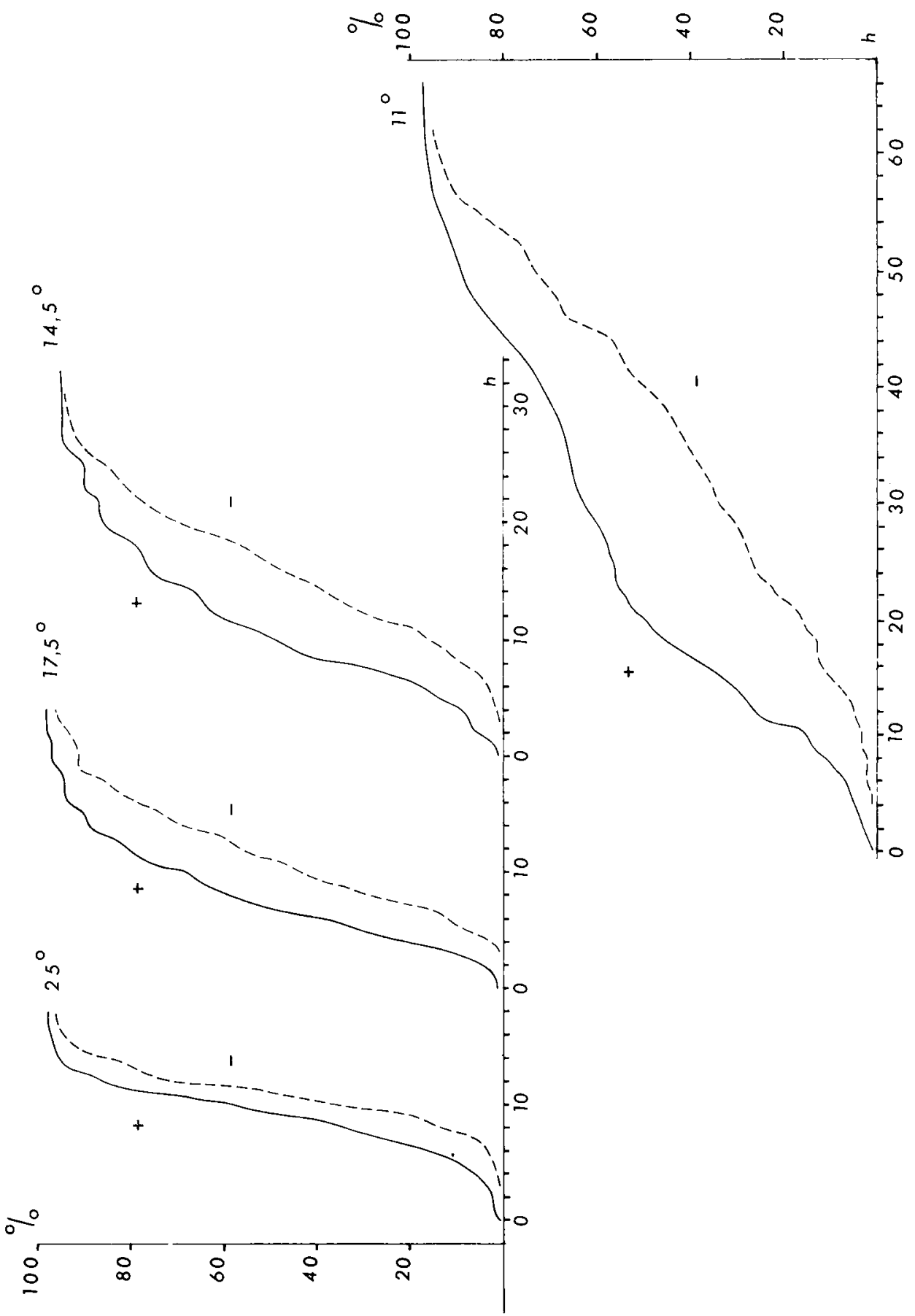

FIG. 3. - Germination des semences à différentes températures (Variété "SV 12 》)

Pour chaque essai : $+=\operatorname{lot}+\mathbf{A b},-=\operatorname{lot}-\mathbf{A b}$; les premières germinations (point 0 en abscisse) débutent respectivement après l'ensemencement dans les délais de $24 \mathrm{~h} 30,36 \mathrm{~h} 30,47 \mathrm{~h} 15$ et $73 \mathrm{~h} 45$ pour les températures de $25,17,5,14,5$ et 11

Aвв. 3. - Keimen der Samen bei verschiedenen Temperaturen (Varietät "SVI2 》)

Für jeden Versuch bedeutet $+=$ Parzelle $+\mathbf{A b} ;-=$ Parzelle - Ab. Die Erstkeimungen (Punkt $\mathbf{O}$ auf der Abszisse) erfolgen jeweils nach der Aussaat in Abständen von 24 1/2 Stdn, $361 / 2 \mathrm{Stdn}, 47 \mathrm{1} / 4 \mathrm{Stdn}$, $733 / 4$ Stdn bei Temperaturen von $25^{\circ} \mathrm{C}, 17,5^{\circ} \mathrm{C}, 14,5^{\circ} \mathrm{C}$ und $11{ }^{\circ} \mathrm{C}$. 


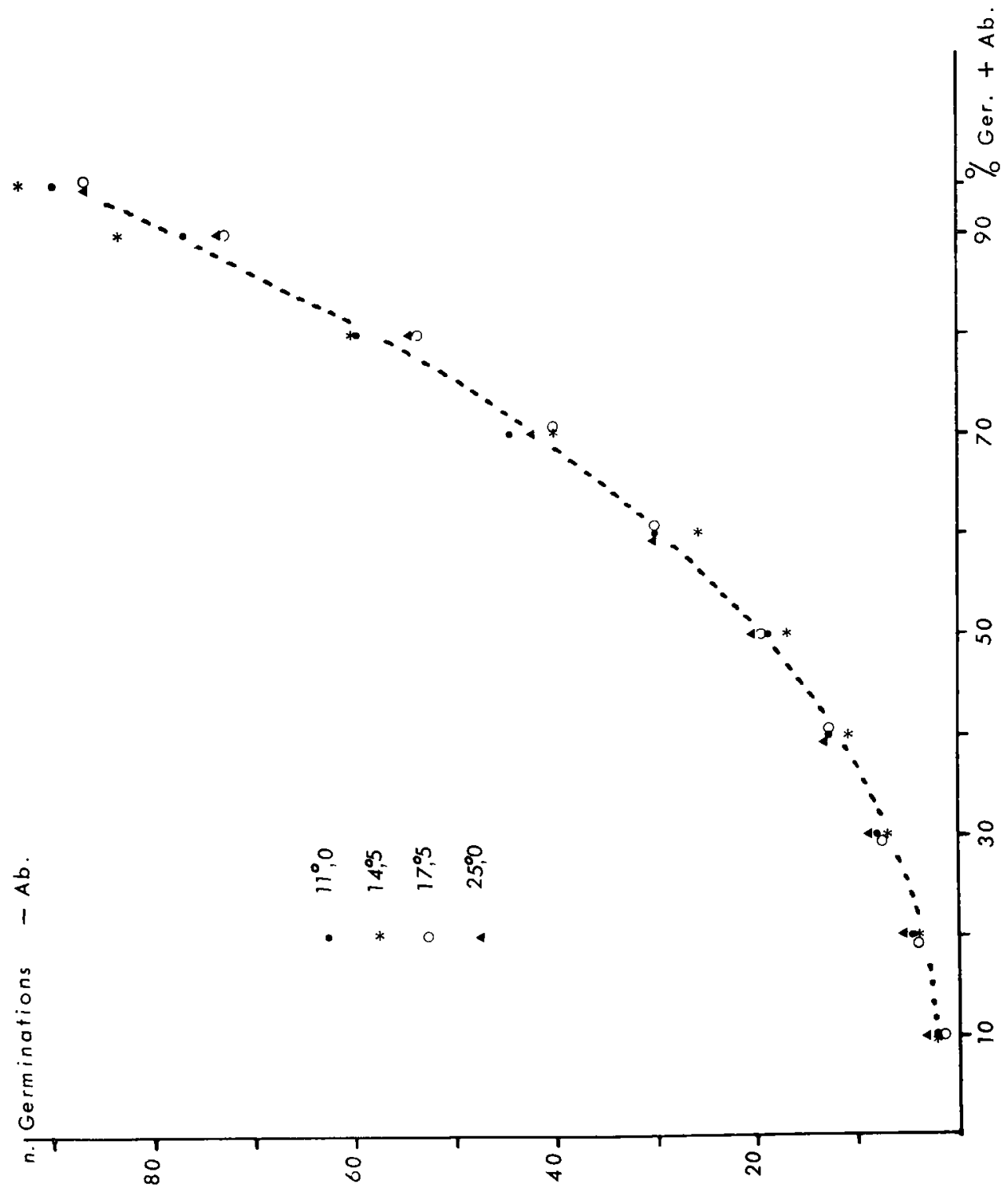

FIG. 4. - Energie germinative des semences autogames (en ordonnée) par rapport à celle des semences allogames (en abscisse). La diffërence d'énergie n'est pas influencée par la tempêrature (Variété «SV 12 »)

Авв. 4. - Keimfähigkeit der autogamen Samen (Ordinate) im Verhältnis zu der der allogamen (Abszisse). Der Unterschied in der Keimfähigkeit ist nicht temperaturbedingt 
des lots $-\mathrm{Ab}$ et des lots $+\mathrm{Ab}$, sont identiques pour chaque moment de l'évolution de la germination sans être influencées par la température.

\section{III. - DISCUSSION}

\section{A. - Utilisation des cages}

Les effets de la présence de la toile métallique sur les conditions climatiques existant sous la cage ont été examinés antérieurement. BarBiER (1962) enregistre une faible différence de l'insolation et de l'agitation de l'air; RuBIs et al. 1966 notent que les cages ont une action faible, pas ou peu significative.

Dans le cas présent les lots + Ab et PA ont en commun la visite des abeilles. Si le butinage du lot + Ab était important, celui du lot PA l'était tout autant du fait de la présence d'un rucher à faible distance et de l'absence presque absolue d'autres sources de nectar; de plus le lot PA bénéficiait de la visite de nombreux hyménoptères très actifs, appartenant à plusieurs genres et espèces, qui n'ont pas été déterminés. Butler et al. (1966) citent pour l'Arizona 40 espèces d'hyménoptères pollinisateurs chez le carthame. Dans ces conditions le lot en plein air se trouve, en ce qui concerne la pollinisation, dans une situation semblable à celle du lot $+\mathrm{Ab}$ mais les plantes de ce lot intègrent dans leurs semences les différences climatiques que la présence de la cage peut provoquer. Or dans la figure 2 la position des plantes des deux lots est confondue en un nuage commun. L'analyse statistique, appliquée aux teneurs en huile et aux poids moyens des semences, ne fait apparaître aucune différence pour les teneurs; quant aux poids, la faible différence qui existe n'est pas significative, le $\mathrm{F}$ calculé est de 3,39 alors que les tables indiquent à $\mathrm{P} .0,05 \mathrm{~F}=$ 3,97 et à $\mathrm{P} .0,01 \mathrm{~F}=6,98$.

Les pieds de carthame isolés sous les cages sont placés dans des conditions rigoureusement comparables sauf en ce qui concerne le mode de pollinisation, aussi toute différence significative ne peut être attribuée qu'au facteur pollinisation. Pour le lot $+\mathrm{Ab}$ il s'agit essentiellement d'une pollinisation entomophile très intense, les abeilles, ne disposant que des fleurs de carthame de la cage, les visitent très activement et transportent le pollen de fleur en fleur. Compte tenu de ce mode de butinage et du décalage de maturité sexuelle des organes mâles et femelles de la fleur, il est évident que le pollen déposé sur un stigmate provient exclusivement d'une ou beaucoup plus probablement de nombreuses autres fleurs; dans ces conditions la fécondation est en très grande proportion allogame.

Pour les plantes de la cage - Ab par contre, l'aspect général de la floraison montre bien que le mode de pollinisation est totalement différent de celui réalisé par les abeilles. Le pollen qui atteint le stigmate ne provient, lorsque 
le stigmate devient réceptif, que des étamines de la même fleur, mais pendant le temps écoulé il est à craindre que le pollen ait perdu une partie de sa vitalité; des essais non publiés de germination du pollen de luzerne (Medicago sativa L.) nous ont montré que les grains de pollen exposés aux rayons du soleil perdent rapidement leur pouvoir germinatif. Le pollen peut aussi provenir des autres fleurs du même capitule et plus rarement encore des fleurs d'un autre capitule de la même plante; dans ces cas si la pollinisation est croisée il s'agit toujours d'une autofécondation sur le plan génétique. Toutefois on ne peut écarter la possibilité d'une pollinisation, sous l'action du vent, par frottement de deux capitules appartenant à des plantes différentes. De même on ne peut refuser l'éventualité, fort peu probable, de la présence d'un apoïde terricole né sous la cage après le traitement insecticide.

Le fait que sur 38 essais de germination celle-ci ait débuté en même temps dans 5 essais chez les lots $+\mathrm{Ab}$ et $-\mathrm{Ab}$ indique que des pollinisations croisées ont pu effectivement se produire chez les lots - $\mathbf{A b}$, toutefois les semences à germination rapide présente dans ce lot sont en nombre très réduit ( 1 à 2 au plus), et ne peuvent pas modifier sensiblement la courbe qui reprend aussitôt sa forme normale.

\section{B. -- Production des semences}

Dans tous les cas on constate (tableau 1 et 2) que la pollinisation par les insectes provoque la formation de semences plus petites, mais celles-ci étant plus nombreuses la production du lot ou d'une plante est toujours plus abondante.

Cozin (1966) remarque une augmentation de poids de la semence chez plusieurs variétés de lupin grâce à la pollinisation croisée, cependant que RuBrs et al (1966) sur le carthame Barbier et Abid (1966) sur le tournesol, White (1972) sur le colza notent que le poids moyen des semences produites par les plantes soumises à une pollinisation entomophile est plus faible qu'en autopollinisation, mais le fait est particulièrement apparent sur la figure $n^{0} I$ où on peut calculer pour les lots $+A b$ et $P A$ que le pic de la courbe qui couvre 8 classes ( 9 à 16$)$ comprend respectivement 86 et $90 \%$ des semences alors que pour la partie la plus élevée de la courbe du lot $-\mathrm{Ab}, 9$ classes ne représentent que $59 \%$ des semences (classes no 12 à 20). De ces remarques il ressort que les semences des lots $+\mathrm{Ab}$ sont plus intéressantes pour les semailles que celles des lots - Ab, car elles sont plus nombreuses à l'unité de poids; (voir tableau 2 et 3$)$.

De leur côté Panov et Petcov (1973) constatent sur poirier une corrélation très nette entre le nombre de visites des abeilles sur les fleurs, le taux de nouaison, le poids moyen des fruits et le nombre des pépins. Pour les plantes à fruits charnus il est bien connu qu'une bonne pollinisation provoque au 
contraire la formation de fruits plus volumineux; Free (1970) et Come (1968) citent de nombreux travaux indiquant que le volume du fruit est en relation avec la présence des graines. Cette relation est évidente chez la pomme (Kurennoi, 1969), chez la clémentine (BARBIEr 1964), chez la poire (Schänder (1955) citée par FREE). La présence des pépins active la maturation de la clémentine (BARBIER, 1964) et même permet un jaunissement plus rapide, comme elle diminue les pertes d'eau de la pomme après la récolte (Come, 1968). Ce dernier constate que c'est beaucoup plus la vitalité des graines (estimée par leur aptitude à germer) que leur nombre qui intervient et il considère que par une meilleure pollinisation on peut espérer augmenter le nombre et la vitalité des graines. Or Bonfante (1972) montre que les abeilles transportant du pollen de la variété de pomme c Stark delicious » pendant deux jours seulement au cours de la floraison de c Golden delicious ) ont notablement augmenté le nombre des pépins dans les fruits.

Pour les fruits charnus, la nouaison et la formation des graines sont directement liées à la pollinisation, alors que le développement du fruit, sa maturation ainsi que sa conservation même après la récolte, apparaissent comme des phénomènes secondaires à cette pollinisation. Pour le tournesol, le colza et le carthame la plus grande quantité de semences formées ainsi que le plus faible poids moyen de celles-ci, sont en relation directe avec la pollinisation réalisée par les insectes.

\section{C. - Teneur et production d'huile}

Au premier abord les semences des lots en allopollinisation ne semblent pas prédisposées à une forte production d'huile, car à un format réduit correspondent une plus faible proportion d'amandes, contenant l'huile et une plus forte proportion de téguments ne contenant pas d'huile, bien que TouboL et Kaparis (1965) décèlent une faible quantité d'huile dans les enveloppes de semence de tournesol. Or, il est constaté ici, en accord avec RuBIS D.D. et al (1966) sur le carthame, Kamennobrodskay (1963) et BARBier et al (1967) sur le tournesol que si les lots $+\mathrm{Ab}$ et $\mathrm{PA}$ présentent entre eux une extrême analogie, ils présentent en même temps une grande différence avec le lot Ab (tableau 1 et 2 - fig. 2).

Cette différence, confirmée par l'analyse statistique (tableau 3) est incontestablement due au mode de pollinisation. De telles constatations impliquent que l'allogamie favorise l'accumulation de l'huile dans les amandes dans une grande proportion puisque les amandes sont plus petites. BARBIER et al (1967) calculent sur tournesol que chez les semences du lot + Ab l'amande représente $71,4 \%$ du poids de la semence contre $76,0 \%$ pour les semences du lot $-\mathrm{Ab}$. Dans ces conditions la différence de teneur en huile des semences entières qui est $7,6 \%$ atteint pour les amandes seules $13,9 \%$. 
TABL. 3. - Analyse de variance des teneurs individuelles en huile des lots $+A b$ et $-A b$

TAB. 3. - Varianzanalyse des individuellen Oegehaltes der Parzellen $+A b$ und $-A b$

\begin{tabular}{|c|c|c|c|c|}
\hline $\begin{array}{l}\text { Variance } \\
\text { Varianz }\end{array}$ & $\begin{array}{l}\text { Somme des } \\
\text { carrés des écarts } \\
\text { Summe d. Quadrate } \\
\text { d. Abweichungen }\end{array}$ & D.L. & $\begin{array}{l}\text { Variance } \\
\text { Varianz }\end{array}$ & $\mathbf{F}$ \\
\hline$\underset{\text { gesamt }}{\operatorname{Totale}} \ldots \ldots \cdots \cdots$ & 179,831 & 76 & & \\
\hline $\begin{array}{l}\text { entre groupes ..... } \\
\text { zwischen d. Gruppen }\end{array}$ & 77,522 & I & 77,522 & \\
\hline $\begin{array}{l}\text { erreur } \ldots \ldots \ldots \ldots \\
\text { Fehler }\end{array}$ & 102,309 & 75 & 1,364 & 56,830 \\
\hline
\end{tabular}

$F$ des tables $-0,05=3,97$. $-0,01=6,98$.

Pour le carthame nous ne possédons malheureusement plus toutes les données pour procéder à la même comparaison ${ }^{1}$ ). Seule une analogie avec les résultats obtenus sur tournesol peut combler cette lacune. On remarque alors au tableau 4 que la teneur en huile des amandes est beaucoup plus élevée

TaBL. 4. - Teneur en huile des amandes isolées : Variété « SV 12 »

TAв. 4. - Oelgehalt der geschälten Samen (Varietät « SV 12 »

\begin{tabular}{|c|c|c|c|}
\hline & $-\mathbf{A b}$ & P.A. & $\begin{array}{l}\text { Différence } \\
\text { Differenz }\end{array}$ \\
\hline $\begin{array}{l}\text { Teneur en huile des semences entières en } \% \text { de la matière sèche .. } \\
\text { Oelgehalt der Gesamtsamen in \% } \% \text { d. Trockenmasse }\end{array}$ & 26,70 & 28,72 & 2,02 \\
\hline $\begin{array}{l}\text { Proportion de l'amande dans la semence entière en } \% \ldots \ldots \\
\text { Anteil d. Samenkerne im Gesamtsamen in } \%\end{array}$ & 149,10 & 44,02 & \\
\hline $\begin{array}{l}\text { Teneur en huile des amandes isolées }{ }^{2} \ldots \ldots \ldots \ldots \ldots \ldots \ldots \ldots \ldots \\
\text { Oelgehalt der geschälten Kerne }{ }^{2}\end{array}$ & ${ }^{1} 54,37$ & 65,24 & 10,87 \\
\hline
\end{tabular}

1. Valeurs obtenues par le calcul.

2. Sans tenir compte de l'huile qui pourrait être apportée par les enveloppes des semences.

1. Werte errechnet.

2. Ohne Berücksichtigung des Oels, das möglicherweise von den Samenhüllen beigesteuert werden könnte.

(1). Les pesées faites sur les semences du lot en plein air étant seules en notre possession. 
que celle des semences entières et que la différence entre les teneurs passe de 2,02 à 10,87 . Malgré une certaine réserve qui s'attache à ces calculs, on voit que la grande augmentation de différence de teneur est due à la forte proportion des enveloppes dans le poids de la semence. On remarque aussi que les augmentations de teneurs des amandes isolées de carthame et de tournesol sont assez voisines.

La productivité du carthame est sensiblement influencée par la pollinisation entomophile; la variété “ Gila », dont la productivité est la plus élevée, est également la plus sensible à ce facteur.

\section{D. - Germination des semences}

En 1966 l'un d'entre nous (BARBiER non publié) remarque que les semences de vesce fourragère (Vicia sativa L.) et de tournesol obtenues en pollinisation entomophile germent plus rapidement que celles formées en autopollinisation. Le grand nombre de semences de carthame de la variété “ SV 12 " ainsi que les appareillages dont nous disposions permirent de reprendre au printemps 1973 les observations dont les résultats constituent l'essentiel des figures 3 et 4 .

Il est remarquable de constater que le mode de pollinisation du carthame, qui reste sans effet sur le taux final de germination ou faculté germinative, intervient fortement sur deux particularités de l'énergie germinative des semences.

$1^{\circ}$ les semences des lots $+\mathbf{A b}$ réagissent rapidement aux conditions favorables du milieu et les premières germinations sont constatées avec une avance de 3 à 6 heures sur celles des semences des lots - Ab.

$2^{\circ}$ L'évolution elle-même des courbes des lots $+\mathrm{Ab}$ est plus rapide. Ainsi l'allogamie détermine chez la semence une énergie germinative plus grande que celle qui résulte de l'autogamie. La différence d'énergie constatée au cours des essais de germination apparaît être indépendante de la température. A cet égard la figure $n^{0} 4$ montre qu'aux diverses températures utilisées, les lots - Ab présentent des taux de germination très voisins entre eux en même temps que très inférieurs à ceux des lots $+\mathrm{Ab}$. La différence étant de l'ordre de 20 à 30 unités pour des germinations comprises entre 30 et $80 \%$ chez les lots $+\mathrm{Ab}$.

Compte tenu des constatations faites ici sur le poids des semences et sur leur énergie germinative, les semences autogames, moins nombreuses à l'unité de poids et possédant une énergie germinative plus faible, présentent une valeur culturale nettement inférieure à celle des semences allogames.

Au sujet de l'énergie germinative des semences il est intéressant de relever que Lala Urie (1957) constate que chez le chardon béni (Cnicus benedictus L) les semences les plus lourdes germent moins vite que celles de poids faible, et 
il suppose qu'en fin de cycle végétatif les semences formées n'ont pas le temps d'arriver à maturité, mais par analogie avec ce qui vient d'être observé sur le carthame on peut envisager aussi un défaut de pollinisation.

Par ailleurs Radchenko (1964) sur colza puis Cozin (1966) sur lupin semblent avoir été les premiers à signaler la relation existant entre la vitesse de germination des graines et la pollinisation entomophile sans toutefois apporter à cette observation tout l'intérêt qu'elle mérite.

De leur côté BenedeK et GraAl (1972) suivent pendant 8 jours la germination des semences d'oignons (Allium cepa L.), en opérant dans des boîtes de Petri garnies de papier buvard humide et à la température de 20 à $22^{\circ}$. Ils montrent qu'il existe une différence hautement significative en faveur de la pollinisation entomophile, toutefois les courbes publiées rappellent celles que nous avions obtenues au cours d'essais antérieurs la température et l'humidité du substrat ainsi que celles de l'air n'étaient pas rigoureusement contrôlées; de plus les comptages effectués une fois par jour ne premettent pas de constater s'il existe un retard dans les premières germinations des semences obtenues en dehors de l'intervention des abeilles.

En définitive la plus grande énergie germinative des semences formées sous l'action de la pollinisation entomophile qui a été signalée sur diverses plantes et démontrée sur oignon et carthame, apparaît être une manifestation de l'hétérosis, ou " stimulation hétérozygotique ", tel que Shull (1914) a défini ce terme (d'après BERNINGER 1960), puisqu'elle s'applique à la graine elle-même c'est-à-dire à la nouvelle plante formée.

Toutefois en ce qui concerne les différences de grosseur et de teneur en huile des graines qui sembleraient beaucoup plus dépendre de la plante mère, on doit savoir que Nitsh (1954) a montré que les tubes polliniques, même s'ils arrivent au niveau de l'ovule après que la fécondation soit effectuée, apportent des auxines favorables au développement de la graine et à sa nouaison en même titre que celles émises par l'embryon. Or la pollinisation entomophile provoque l'apport sur le stigmate d'un plus grand nombre de grains de pollen que l'autopollinisation, de plus ces pollens de par leur origine étrangère à la plante, germent plus facilement sur le stigmate et l'allongement des tubes polliniques à travers le style est également favorisée, il en résulte que la quantité d'auxines polliniques doit être nettement plus importante dans le cas d'une pollinisation entomophile.

Dans quelle mesure ces auxines polliniques interviennent-elles sur la grosseur de la graine et sur sa teneur en huile? On peut émettre l'hypothèse que leur action si elle n'est pas de courte durée est rapidement dépassée par celle des duxines produites par l'embryon et qu'en fin de compte l'abondance de ces dernières, favorisée par l'état hétérozygotique déterminerait la grosseur de la graine et la teneur en matière grasses de l'amande, elle serait ainsi la 
première manifestation de l'hétérosis alors que la nouaison serait favorisée par les auxines d'origine pollinique.

\section{CONCLUSION}

Les diverses observations effectuées sur la carthame démontrent que le mode de pollinisation a des conséquences importantes sur le potentiel de reproduction des plantes et sur les qualités des semences.

Certains phénomènes, comme l'augmentation du nombre de semences, la diminution de leur poids et l'accroissement de leur teneur en huile sous l'action de la pollinisation entomophile se trouvent amplement vérifiés sur le carthame, en même temps qu'une observation confirme et précise les modalités d'une manifestation peu connue de l'hétérosis sur l'énergie germinative des semences.

Les effets de la pollinisation entomophile et de la fécondation croisée présentent un certain intérêt sur le plan cultural pour la production de graines de semence et la production d'huile. Mais sur le plan biologique les observations faites ici sur l'énergie germinative des semences apportent un argument important pour appuyer la thèse selon laquelle la reproduction des plantes et la survie des espèces sont étroitement liées à la faune entomophile qui reste l'agent indispensable de l'allogamie. Cette remarque est valable, même pour des plantes qui, comme la carthame, présentent un taux élevé d'autofertilité et d'autopollinisation.

Reçu pour publication en octobre 1975.

Eingegangen im Oktober 1975.

\section{REMERCIEMENTS}

Nous remercions MM. Courtin et Surrugue de l'Institut national de la Recherche agronomique du Maroc ainsi que M. Cazote du projet Pram pour l'aide qu'ils ont bien voulu nous apporter pour la réalisation des essais aux champs. Nous remercions également M. Audemard du C.R.A. du Sud-Est pour ses conseils et M. Come du C.N.R.S. à Meudon pour le grand intérêt qu'il a porté à ce travail.

\section{ZUSAMMENFASSUNG}

In den Ländern mit mediterranem Klima und geringen Niederschlägen kann der Echte Saflor eine interessante Quelle fetthaltiger Substanzen sein. Beim Anbau erreicht die Pflanze eine Höhe von 1,50 m und trägt durchschnittlich $30 \mathrm{Köpfchen} \mathrm{zu} \mathrm{je} \mathrm{40-70} \mathrm{gelben} \mathrm{Blüten.} \mathrm{Die}$ Verkümmerungsrate ist stets sehr hoch.

Die Bestäubungsversuche wurden auf $36 \mathrm{~m}^{2}$ grossen Flächen durchgeführt (PA $=$ Kontrolle unter freiem Himmel, $+\mathrm{AB}=$ unter Drahtgitter mit einem Bienenvölkchen, $-\mathrm{AB}=$ unter Drahtgitter ohne jedes Insekt). 
Mit beginnender Blütezeit welken die Blüten der Parzellen PA und $+\mathrm{Ab}$ während 24-48 Stunden und werden schnell braun, während die Blüten der Parzelle $-\mathrm{Ab}$ eine Woche lang blühen und sich nur langsam verfärben.

Die ins einzelne gehenden Untersuchungen der Ernten und der Samen erlauben folgende Feststellungen :

1. der Echte Saflor ist autogam und zwar je nach Varietät in verschiedenem Grad; aber die Allogamie gewährleistet eine ergiebigere Samenbildung und beeinflusst gleichzeitig in verschiedener Weise die Samenqualität.

2. Durchschnittsgewicht. Die allogamen Samen sind leichter als die autogamen. Der Vergleich der Kurven, die die Verteilung der Samen nach ihrem Gewicht angeben, zeigt, dass die allogamen Samen sich um ein mittleres Gewicht von $38,5 \mathrm{mg}$ gruppieren, während die autogamen Samen eine diffusere Verteilung aufweisen und deutlich nach Durchschnittsgewichten hin tendieren, die höher als $43,8 \mathrm{mg}$ liegen (Abb. 1).

3. Der Oelgehalt. Der für jede untersuchte Pflanze festgestellte Oelgehalt zeigt, dass der Einfluss der Drahtgeflechte praktisch gleich Null ist. Bei den Parzellen PA und + Ab liegen die Werte des Oelgehaltes dicht beieinander; sie betragen im Mittel 28, $72 \%$, während die Werte bei den Pflanzen der Parzelle - Ab eine deutliche Streuung aufweisen. Der Oelgehalt beträgt hier durchschnittlich 26, $72 \%$, was einen Unterschied von $2 \%$ bedeutet (Abb. 2).

Die Bestimmung der Fettstoffe, die an den von der Samenschale befreiten Kernen durchgeführt wurde, ergab einen gewissen Unterschied des Oelgehaltes : $65,24 \%$ gegenüber $54,37 \%$, also einen Unterschied von $10,87 \%$.

4. Das Keimen der Samen. Unter streng kontrollierten Bedingungen ergab sich :

- Die allogamen Samen beginnen stets zuerst zu keimen.

- Die allogamen Samen haben eine viel stärkere Keimfähigkeit als die autogamen (Abb. 3).

- Der Unterschied in der Keimkraft ist nicht temperaturbedingt (Abb. 4).

- Die Keimfähigkeit ist die gleiche, einerlei welcher Art die Bestäubung war.

Diese Tatsachen sind die ersten Bestätigungen der Heterosis; sie bezeugen die Überlegenheit der Allogamie über die Autogamie und erbringen den Beweis dafür, dass selbst für eine Pflanze, bei der Selbstbestäubung möglich ist, die Vermehrung von der sie umgebenden lebendigen Umwelt abhängig ist.

\section{RÉFÉRENCES BIBLIOGRAPHIQUES}

BarBier E., 1962. Quelques facteurs de la productivité quantitative et qualitative des essences chez les lavandes. Thèse Fac. Sci. Paris.

Barbier E., 1964. Pollinisation et fructification du clémentinier. Ann. Abeille 7 (1) : 63-80.

Barbier E., Abid M., 1966. Pollinisation et formation des graines chez le tournesol (Hélianthus annuus L.) El Awamia $21: 61-83$.

Barbier E., Berrada A., Straub J.-M., 1967. Effets du butinage des abeilles sur la teneur en matières grasses du tournesol. El Awamia 22 : 1-12.

Benedek P. et GraAl E., 1972. The effect of insect pollination on seed onion, with observations on the behaviour of honeybees on the crop. J. Apic. Res. 11 (3) : 175-180.

Berninger E., 1960. Note sur quelques aspects de l'hétérosis. Ann. Amelior. Plantes 10 (3) 34.5350 .

Bonfante S., 1972. Un distributeur de pollen et ses différents emplois. Bull. Sci. Apimondia, p. $173-175$.

Butler G.D. Jr., Werner F.G. et Levin M.D., 1966. Native bees associated with safflower in south central Arizona. J. Kansas entomol. Soc. $39: 434-436$. 
COME D., 1968. Influence des graines sur la transpiration et l'évolution de la coloration des pommes après la récolte. Congr. Nat. Soc. Savantes. 93. 1968. Tours, t. III p. 647-659.

Соме D., 1970. Les obstacles à la germination. Masson et Cie - Paris.

Cozine R.B., 1967. Influence de la pollinisation du Lupinus par les Abeilles sur la production des semences et leur qualité. Congr. int. Apic. 21. 1967. Maryland (USA) : 530-531.

Free J.B., 1970. Insect pollination of crops. Academic Press-London.

Kamennobrodskaya V., 1963. Influence de la pollinisation par les abeilles sur la qualité des graines de tournesol (en Russe). Pchelovodstvo, 40 (7) : 21.

Kunenvor N.M., 1969. Importance de l'abeille comme facteur régulateur de la productivité du pommier. Congr. int. Apic., 22. 1967. München. 481-484.

Lalaurie M., 1957. Germination des akènes. Relation avec le poids et la taille. Société Pharmacie, Montpellier, 138-146.

Nitsch J.R., 1954. Auxines et croissance des fruits. Congr. int. Bot. 8. Sect II 360-367.

Panov V., Petcov V., 1973. Activité pollinisatrice des abeilles sur le poirier. Congr. int. Apic. 24. 1973. Buenos Aires, 466-468.

Radchenko T.G., 1964. Influence de la pollinisation sur la récolte et la qualité des graines de colza (en Ukrainien) Bdzhil'nitstvo (1) : 68-74 (in Apic. Abst. $n^{0} 380 / 69$ ).

Rubis D.D., Levin M.D. et Mc Gregor S.E., 1966. Effects of honey bee activity and cages on attributes of thin-hull and normal safflower lines. Crop Sci. 6 : 11-14.

Toubol V. et Kaparis V., 1965. Matières grasses dans les coques des graines de tournesol. El Awamia (15).

White B., 1972. La pollinisation des cultures oléagineuses et l'effet des pesticides utilisés sur celles-ci. Bull. Sci. Apimondia : 428-435. 